\title{
Effectiveness of measuring tension during arthroscopic rotator cuff repair
}

\author{
Shin Yokoya ${ }^{*}$, Yoshihiro Nakamura, Yohei Harada, Hiroshi Negi, Ryosuke Matsushita, Norimasa Matsubara, \\ Yasuhiko Sumimoto and Nobuo Adachi
}

\begin{abstract}
Purpose: Arthroscopic rotator cuff repair (ARCR) for relatively small rotator cuff tears (RCTs) has shown promising results; however, such surgery for larger tears often results in failure and poor clinical outcomes. One cause of failure is over-tension at the repair site that will be covered with the tendon stump. Reports on the clinical outcomes using ARCR with tension $\leq 30 \mathrm{~N}$ are lacking. This study aimed to evaluate ARCR outcomes and failure rates using less tension $(30 \mathrm{~N})$ and to assess the prognostic factors for failure.

Methods: Our study group comprised of 118 patients who underwent ARCR for full-thickness RCTs with full tendon stump coverage of the footprint with a tension of $\leq 30 \mathrm{~N}$, measured using a tension meter; no additional procedures, such as margin convergence or footprint medialisation, were performed. The failure rate was calculated, and the prognostic factor for failure was assessed using multivariate regression analyses.

Results: There were seven cases of failure in the study group. Postoperatively, flexion and internal rotation ranges of motion, acromiohumeral interval, muscle strength, and clinical results improved significantly. Using multivariate regression analyses, intraoperative concomitant subscapularis tendon lesion and pre-operative infraspinatus tendon retraction, assessed using radial-sequence magnetic resonance imaging, were significantly correlated with post-ARCR failure using less tension ( $p=0.030$ and $p=0.031$, respectively).
\end{abstract}

Conclusion: ARCR is likely to succeed for RCTs that can be extracted using tension $\leq 30 \mathrm{~N}$. However, cases with more severe subscapularis tendon lesions and those with high infraspinatus tendon retraction may show surgical failure.

Level of evidence: LEVEL IV Retrospective case series

Keywords: Rotator cuff tear, Arthroscopic rotator cuff repair, Repair tension, Failure rate, Tension meter, Prognostic factor

\section{Background}

Rotator cuff tears (RCTs), often occurring in older people, are a possible cause of shoulder pain. When first-line conservative therapy is ineffective, arthroscopic rotator cuff repair (ARCR) is widely performed. Excellent results have been reported using ARCR for relatively small RCTs; however, in patients with larger tears, ARCR has

\footnotetext{
*Correspondence: yokoyan822@msn.com
}

Department of Orthopaedic Surgery, Graduate School of Biomedical and Health Sciences, Hiroshima University, Kasumi 1-2-3, Minami-ku, Hiroshima, Japan frequently resulted in failure and poor clinical outcomes $[4,8,12,32]$. One cause of failure has been considered to be due to over-tension at the repair site. Davidson and Rivenburgh reported poor clinical results when tears were repaired under a tension of $>8 \mathrm{lb}$ (approximately $35 \mathrm{~N}$ ) [5]. Therefore, we developed a method of muscle advancement by elevating the supraspinatus and infraspinatus muscles from the scapula and advancing these muscles laterally when the tension at the repair site was $>30 \mathrm{~N}$ based on previous studies $[5,18,25,36]$ and reported a lower ARCR failure rate in patients using this advanced procedure [36]. However, it is unknown to what 
extent clinical results and failure rates could be improved using ARCR with less tension, that is, at $\leq 30 \mathrm{~N}$. Kim et al. reported that the tension on the repair site in one treatment group had resulted in post-ARCR failure when using significantly higher tension than that in a healed group [18]; however, reports concerning the results of cuff integrity and clinical outcomes using ARCR with tension $\leq 30 \mathrm{~N}$ are lacking. We aimed to evaluate the outcomes and failure rates of ARCR with tension $\leq 30 \mathrm{~N}$ and analyse the prognostic factors causing failure after ARCR under such conditions. The hypothesis was that repairing with a tension of less than $30 \mathrm{~N}$ would reduce failure after ARCR, and the larger tears including multiple tendons and the more degenerated RCTs would be the prognostic factors.

\section{Materials and methods}

These retrospective case seris were approved by our Institutional Review Board (permission number: E-2079). ARCR was performed using the suture bridge technique for 349 consecutive patients diagnosed with RCTs between October 2010 and June 2018, who could be followed up for at least 2 years. Patients with isolated subscapularis (SSC) tendon tears, osteoarthritis or rheumatoid arthritis, and/or partial-thickness RCTs or those requiring surgical revision after a failed repair were excluded from the current study. During this period, no patient had post-operative infection. No medialisation of the footprint [9], margin convergence [2], and interval slide [33] were added for these patients. Furthermore, patients who had undergone ARCR with partial repair [19], muscle advancement [36] or any augmentation, including autograft [24], allograft [10], artificial biomaterial [11, 35, 37, 38], or bone marrow [6, 38] were also excluded. Finally, a total of 118 patients who had undergone ARCR with a bridging suture technique measuring a tension of $\leq 30 \mathrm{~N}$ with full coverage of the footprint by the tendon stump, as measured using a tension meter (Ligament tensioner 3, MEIRA Corporation, Nagoya, Japan, Fig. 1), between October 2011 and June 2018 were enrolled in the current study. During this period, when the measured tension exceeded $30 \mathrm{~N}$, we performed ARCR with muscle advancement to extract the retracted tendon [36] and/or with artificial biomaterial (polyglycolic acid sheet $[37,38]$ ) reinforcement to the original footprint without any medialisation to enhance the healing capacity [35]; patients who underwent these procedures were excluded from the present study. In the current study, the average age was $66.1 \pm 9.4$ (range, $25-82$ ) years. The pre-operative data and several surgical treatments are shown in Table 1.

The supraspinatus (SSP) and infraspinatus (ISP) tendon retraction grades, assessed preoperatively using

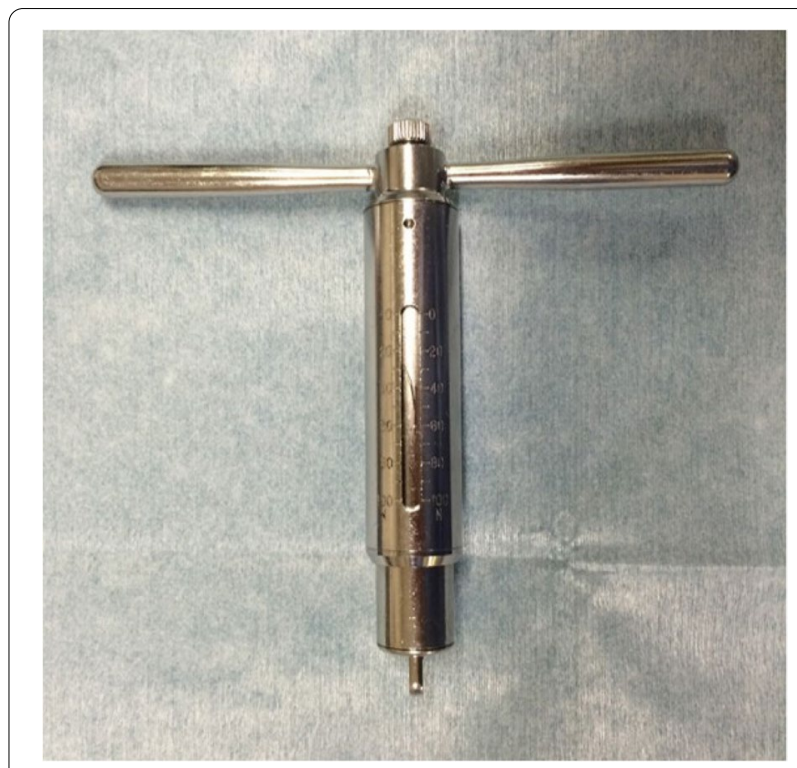

Fig. 1 The tension meter (Ligament tensioner 3, MEIRA Corporation, Nagoya, Japan)

radial-sequence magnetic resonance imaging (MRI) as described by Honda et al. [14], were categorised according to Boileau's classification [31]. The SSC grades and findings were categorised according to the Lafosse classification [20], and the long head of the biceps brachii (LHB) findings was categorised using a modified Lafosse classification [21]. Furthermore, the degree of fatty degeneration (FD) of each rotator cuff muscle was assessed according to the Goutallier classification system [7], and the global fatty degeneration index (GFDI), which was calculated according to the Fuchs report [7].

Table 1 Demographic data

\begin{tabular}{ll}
\hline & $\mathbf{n = 1 1 8}$ \\
\hline Sex, $\mathrm{n}$ & 64 males / 54 females \\
Age, years & $66.1 \pm 9.4$ \\
Affected arm, $\mathrm{n}$ & $85 \mathrm{right} / 33$ left \\
Follow-up period, months & $27.6 \pm 10.3$ \\
Prevalence of DM & $16(+): 102(-)$ \\
SSP tendon retraction & $25 / 69 / 24$ \\
Boileau Stage I/II/II & \\
ISP tendon retraction & $78 / 20 / 14 / 6$ \\
Boileau Stage 0/I/I/III & \\
Concomitant SSC lesion & $46 / 41 / 27 / 2 / 2$ \\
Lafosse type 0/I/I/III/IV & \\
Concomitant LHB lesion & $59 / 27 / 12 / 12 / 8$ \\
modified Lafosse Grade I/II/II/IVN & \\
Fatty degeneration & SSC 65/39/7/6/1 \\
Goutallier classification Grade 0/1/2/3/4 & SSP 27/65/23/3/0 \\
& ISP 61/45/9/2/1 \\
& GFDI 0.76 0.57 \\
\hline
\end{tabular}




\section{Surgical technique}

All surgeries were performed by a single surgeon. ARCRs were performed with the patient under general anaesthesia and placed in the beach chair position with the occasional use of an interscalene block. We first examined the glenohumeral joint in the standard manner and performed a synovectomy. If necessary, a capsular release was also performed. When the intraoperative SSC findings were Lafosse type 2 or higher, the arthroscopic repair was performed. When the intraoperative LHB findings were modified Lafosse grade 2 or higher, tenodesis or tenotomy was performed before SSP and/or ISP repair. We then switched to arthroscopic subacromial space observation and performed subacromial decompression and a cuff release to the maximum extent possible.

\section{Measuring the tension}

Intraoperative tendon excursion has been checked in a $30^{\circ}$ abduction position with a muscle relaxant. At first, the types of rupture, such as crescent-, L-, reverse L-, or U-shaped [3], were checked by reducing the torn cuff tendons with a suture retriever. Between one and three No. 0 nylon threads were applied approximately $1 \mathrm{~cm}$ from the stump of the torn rotator cuff tendon using a suture hook device, depending on the tear shape or size. Usually, one thread was used for an isolated SSP tear, two threads for a combined SSP and anterior section of the ISP tendon tear, and three threads for full-width tendon tears of the SSP to ISP tendons. The nylon threads were retrieved from the antero-lateral portal, and each nylon thread was pulled laterally (Fig. 2) as the torn cuffs were reduced to the footprint originally using a tension meter. The tension required for the tendon stump to cover the entire footprint was then measured. When full coverage

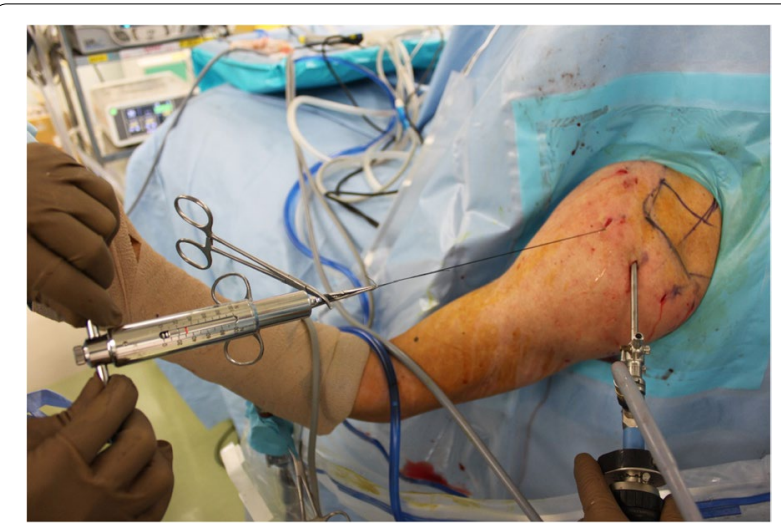

Fig. 2 One to three No. 0 nylon threads are applied to the stump of the torn rotator cuff tendon depending on the tear size and are retrieved from the anterolateral portal. Each nylon is pulled laterally by using a tension meter of the footprint cannot be achieved, even when applying a tension of $\geq 30 \mathrm{~N}$, ARCR with muscle advancement was performed [36]. When full coverage of the footprint was possible with a tension $\leq 30 \mathrm{~N}$ (Fig. 3a, b), ARCR was performed using a suture bridge technique, which we have previously reported as a modified medial double-pulley technique (Fig. 3c) [36]. When there were two medial anchors, one double pulley was created, and when there were three medial anchors, three double pulleys were created. In such cases, there were always two lateral anchors. The sutures from the suture anchors were penetrated at $2 \mathrm{~cm}$ medial to the edge of the torn tendon. If full coverage of the footprint by the tendon stump could be achieved after suture bridge repair completion, the repair was regarded as an anatomical repair. A summary of treatment methods of SSC repair and LHB lesions, with or without capsular release, completion of anatomical repair or not, and bridge configuration pattern is presented in Table 2.

\section{Rehabilitation}

For all patients, the affected arm was immobilised for 6 weeks using an abduction brace. The post-operative rehabilitation protocol was as follows: passive range of motion (ROM) exercise commenced from week one postoperatively and assisted ROM within $90^{\circ}$ abduction commenced from week four. After abduction brace removal, active $\mathrm{ROM}>90^{\circ}$ abduction and isometric muscle strength exercises commenced. The cuff muscle strength exercise commenced from week twelve. When tenotomy or tenodesis of the LHB had been performed, elbow joint ROM was prohibited for 3 weeks. In patients engaging in heavy manual work or sports enthusiasts, elbow joint ROM was permitted 6 months postoperatively.

\section{Evaluation}

Post-operative cuff integrity was assessed using MRI, as described by Sugaya et al. [32]. Types IV and V, according to the Sugaya classification, were regarded as surgical failures. The failure rates were then calculated. The current groups were also sub-divided into healed and failed groups to assess the prognostic factors for ARCR failure. These evaluations were conducted by co-authors blinded about the surgical intervention.

Acromiohumeral interval (AHI) values were measured using pre- and post-operative plain radiographic anteroposterior views in standing and external rotation (ER)/internal rotation (IR) neutral positions, according to the procedure described by Ianotti et al. [15] for each patient. Pre-and post-operative ROM measurements were performed in anterior flexion and ER using a goniometer. The IR value was measured based on the highest 

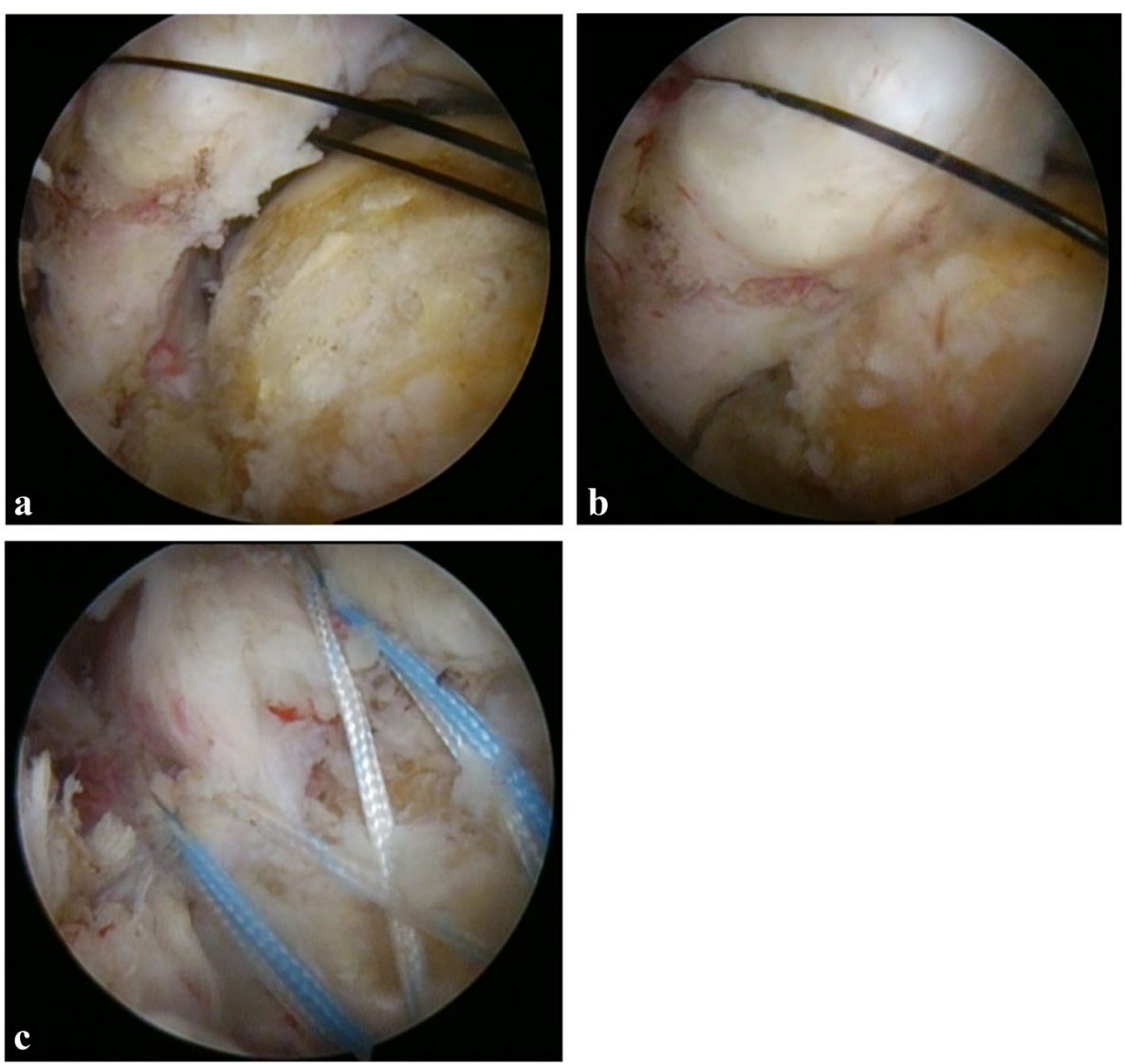

Fig. 3 Intraoperative images of the torn cuff. a Before pulling the nylon thread. b After pulling the nylon thread using a tension of $30 \mathrm{~N}$. $\mathbf{c}$ After repairing the torn cuff using a suture bridge. When full coverage of the footprint is possible with a tension of $\leq 30 \mathrm{~N}$, arthroscopic rotator cuff repair (ARCR) is performed using the suture bridge technique

Table 2 A summary of the surgical treatment

\begin{tabular}{ll}
\hline & $\mathbf{n = 1 1 8}$ \\
\hline SSC treatment & None:55 \\
& Debridement: 13 \\
& Single repair: 24 \\
& Bridge repair: 22 \\
& PMT: 2 \\
& None: 61 \\
LHB treatment & Tenotomy: 38 \\
& Tenodesis: 12 \\
& Rupture: 7 \\
Capsular release & release (+): 34 \\
Anatomical or non-anatomical repair & release (-): 84 \\
Suture bridge configuration & anatomical: 92 \\
& non-anatomical: 26 \\
& $2 \times 2: 108$ \\
& $3 \times 2: 10$ \\
\hline
\end{tabular}

vertebral level that a patient's thumb could reach behind their back for each patient. The pre- and post-operative clinical outcomes were evaluated using the Constant score, the University of California, Los Angeles Shoulder Rating Scale (UCLA) score, and the numeric rating score (NRS) for shoulder pain. The term "pre-operative" refers to the period immediately before surgery, and the term "post-operative" refers to the last follow-up session. The pre- and post-operative AHI, ROM values and the clinical scores were compared between the healed and failed group, respectively.

Additionally, isometric muscle strength of the affected arm on abduction and ER was measured pre- and postoperatively using a hand-held dynamometer (Microfet 2; Nihon Medix Co., Ltd., Chiba, Japan). These strength measurements were performed in a seated position, at a $45^{\circ}$ abduction angle in the scapular plane for abduction strength, and with the upper arm at the side of the body and the elbow in $90^{\circ}$ flexion in an ER/IR neutral position to measure ER strength, as has been previously reported [36]. Each measurement was performed three 
times, and average values were calculated. The muscle strengths were also compared between the healed and failed groups.

To assess prognostic factors for failure in the study group, age, sex, affected side, prevalence of diabetes mellitus (DM), contracture, SSP and ISP tendon retraction, concomitant SSC and LHB injury, AHI, pre-operative ROM and muscle indexes, FD of each muscle, and GFDI were compared between the healed and failed groups, using univariate and multivariate regression analyses.

\section{Statistical analyses}

All values were expressed as means \pm standard deviations. Statistical analyses were performed using the Bell Curve for Excel software (Ver. 3.20; Social Survey Research Information Co., Ltd., Tokyo, Japan). Comparisons between pre- and post-operative values in each group were performed statistically using paired t-test. Comparisons between the healed and failed groups were assessed statistically using Mann-Whitney's U test. Multivariable logistic regression analysis was used to determine prognostic factors for failure after ARCR focusing on the items that had significant differences in univariate analyses and some items previously reported as prognostic factors. Statistical significance was set at a $p$-value $<0.05$.

\section{Results}

We identified 7 (of 118) cases of failure in the current study (failure rate, 5.9\%) (Table 3). In a comparison between the pre- and post-operative values in the healed group, AHI improved significantly in the study group, from $9.4 \pm 1.9 \mathrm{~mm}$ preoperatively to $11.7 \pm 2.2 \mathrm{~mm}$, postoperatively. Regarding ROM, flexion and IR improved significantly after surgery (from $125.9 \pm 34.0$ and $T h 11.7 \pm 2.9$ preoperatively to $157.1 \pm 12.1$ and Th10.0 \pm 2.5 postoperatively $[p<0.001$ and $p<0.001$, respectively]). However, ER did not improve significantly after surgery (from $56.8 \pm 17.1$ preoperatively to $57.2 \pm 16.0$ postoperatively, $p=0.647$ ). The Constant scores, UCLA scores and NRS all improved significantly from $48.4 \pm 14.9,14.8 \pm 4.3$, and $6.6 \pm 1.8$ preoperatively to $83.3 \pm 11.9,32.6 \pm 3.2$, and

Table 3 Cuff integrity and failure rate

\begin{tabular}{lll}
\hline Sugaya classification & $\mathbf{n = 1 1 8}$ & 111 \\
\hline Type I & 49 & \\
Type II & 54 & \\
Type III & 8 & 7 \\
Type IV & 1 & \\
Type V & 6 & \\
Failure rate & $5.9 \%$ & \\
\hline
\end{tabular}

$0.9 \pm 1.3$ postoperatively $(p<0.001,<0.001$, and $<0.001$, respectively). On the other hand, in the failed group, AHI (from $9.4 \pm 2.1$ preoperatively to $11.1 \pm 4.1$ postoperatively, $p=0.098$ ), all ROM (from $139.3 \pm 33.2$, $56.4 \pm 12.8$, and Th12.5 \pm 2.9 preoperatively to $127.9 \pm 35.9,52.9 \pm 12.2$, and $T h 11.7 \pm 2.9$ postoperatively, $p=0.567,0.376$, and 0.486 , respectively), Constant score, and UCLA score (from 54.0 \pm 18.0 , and $17.0 \pm 5.9$ preoperatively to $65.2 \pm 23.3$, and $23.1 \pm 9.2$ postoperatively, $p=0.315$, and 0.140 , respectively) showed no improvement postoperatively, except for the NRS (from $7.0 \pm 2.1$ preoperatively to $3.1 \pm 2.6$ postoperatively, $p=0.013$ ). In the comparison between the healed and failed groups, postoperative flexion angle, Constant score, UCLA score, and NRS had significant differences ( $p=0.003,0.008,0.002$, and 0.005 , respectively) in spite of no significant differences in all preoperative values $(p=0.860,0.193,0.755,0.648,0.288$, 0.342 , and 0.663 , respectively) and postoperative AHI, ROM of ER, and IR ( $p=0.913,0.313$, and 0.846 , respectively). The results of the AHI and ROM and clinical scores are summarised in Table 4.

The abduction and ER muscle strengths in the healed group improved significantly (from $33.1 \pm 17.7$ and $43.0 \pm 18.2$ preoperatively to $68.7 \pm 26.6$ and $65.9 \pm 22.5$

Table 4 Results of the AHI, ROM and clinical scores

\begin{tabular}{|c|c|c|c|}
\hline & $\begin{array}{l}\text { Healed group } \\
(n=111)\end{array}$ & $\begin{array}{l}\text { Failed group } \\
(n=7)\end{array}$ & $p$ value \\
\hline Pre $\mathrm{AHI}, \mathrm{mm}$ & $9.4 \pm 1.9$ & $9.4 \pm 2.1$ & 0.860 \\
\hline Post AHI, mm & $11.7 \pm 2.2$ & $11.1 \pm 4.1$ & 0.913 \\
\hline$p$ value & $<0.001^{*}$ & 0.098 & \\
\hline Pre flexion, ${ }^{\circ}$ & $125.9 \pm 34.0$ & $139.3 \pm 33.2$ & 0.193 \\
\hline Post flexion, ${ }^{\circ}$ & $157.1 \pm 12.1$ & $127.9 \pm 35.9$ & $0.003+$ \\
\hline$p$ value & $<0.001^{*}$ & 0.567 & \\
\hline Pre ER, ${ }^{\circ}$ & $56.8 \pm 17.1$ & $56.4 \pm 12.8$ & 0.755 \\
\hline Post ER, ${ }^{\circ}$ & $57.2 \pm 16.0$ & $52.9 \pm 12.2$ & 0.313 \\
\hline$p$ value & 0.647 & 0.376 & \\
\hline Pre IR, spine level & Th11.7 \pm 2.9 & Th12.5 \pm 2.9 & 0.648 \\
\hline Post IR, spine level & Th10.0 \pm 2.5 & Th11.7 \pm 2.9 & 0.846 \\
\hline$p$ value & $<0.001^{*}$ & 0.486 & \\
\hline Pre constant score & $48.4 \pm 14.9$ & $54.0 \pm 18.0$ & 0.288 \\
\hline Post constant score & $83.3 \pm 11.9$ & $65.2 \pm 23.3$ & $0.008+$ \\
\hline$p$ value & $<0.001^{*}$ & 0.315 & \\
\hline Pre UCLA score & $14.8 \pm 4.3$ & $17.0 \pm 5.9$ & 0.342 \\
\hline Post UCLA score & $32.6 \pm 3.2$ & $23.1 \pm 9.2$ & $0.002 \dagger$ \\
\hline$p$ value & $<0.001^{*}$ & 0.140 & \\
\hline Pre NRS & $6.6 \pm 1.8$ & $7.0 \pm 2.1$ & 0.663 \\
\hline Post NRS & $0.9 \pm 1.3$ & $3.1 \pm 2.6$ & $0.005+$ \\
\hline$p$ value & $<0.001^{*}$ & $0.013 *$ & \\
\hline
\end{tabular}


postoperatively, $p<0.001,<0.001$, respectively), despite no significant differences in the failed group (from $36.9 \pm 21.7$ and $37.1 \pm 21.5$ preoperatively to $38.6 \pm 35.5$ and $54.1 \pm 32.4$ postoperatively, $p=0.872,0.127$, respectively). IR muscle strengths in the healed group also improved significantly (from $77.8 \pm 31.3$ preoperatively to $113.5 \pm 37.1$ postoperatively, $p<0.001)$ despite no significant difference in the failed group (from 71.0 \pm 44.4 preoperatively to $91.1 \pm 44.1$ postoperatively, $p=0.090$ ) Comparison between the healed and failed groups showed no significant differences in all strengths (pre abduction $p=0.405$, pre ER $p=0.547$, pre IR $p=0.392$, post ER $p=0.144$, and post IR $p=0.158$, respectively) except for post-operative abduction strength $(p=0.025)$. The results of the muscle strength indexes are summarised in Table 5.

The results of univariate comparisons between the healed and failed groups in relation to pre-operative assessments in the study group are shown in Table 6 . Only ISP tendon retraction, assessed using radialsequence MRI, and intraoperative concomitant SSC lesion evaluated by Lafosse classification showed significant differences ( $p=0.012$, and 0.006 , respectively). Multivariate regression analysis results also indicated a significant correlation between the pre-operative ISP tendon retraction and concomitant SSC lesion $(p=0.031$ and $p=0.030$, respectively). The odds ratios and $95 \%$ confidence intervals for these variables were 19.4 and 1.31287.3 for ISP tendon retraction, and 4.41 and 1.16-16.8 for the concomitant SSC lesion respectively (Table 7).

\section{Discussion}

This study investigated clinical outcomes and ARCR failure rates and assessed prognostic factors to determine post-ARCR failure using tension $\leq 30 \mathrm{~N}$ to restore

Table 5 Results of isometric muscle strength concerning each group

\begin{tabular}{llll}
\hline & Healed group $(\boldsymbol{n}=\mathbf{1 1 1})$ & Failed group $(\boldsymbol{n}=\mathbf{7})$ & $\boldsymbol{P}$-value \\
\hline Pre AbdS, N & $33.1 \pm 17.7$ & $36.9 \pm 21.7$ & 0.405 \\
Post AbdS, N & $68.7 \pm 26.6$ & $38.6 \pm 35.5$ & $0.025 \dagger$ \\
$p$ value & $<0.001^{*}$ & 0.872 & \\
Pre ERS, N & $43.0 \pm 18.2$ & $37.1 \pm 21.5$ & 0.547 \\
Post ERS, N & $65.9 \pm 22.5$ & $54.1 \pm 32.4$ & 0.144 \\
$p$ value & $<0.001^{*}$ & 0.127 & \\
Pre IRS, N & $77.8 \pm 31.1$ & $71.0 \pm 44.4$ & 0.392 \\
Post IRS, N & $113.5 \pm 37.1$ & $91.1 \pm 44.1$ & 0.158 \\
$p$ value & $<0.001^{*}$ & 0.090 & \\
\hline
\end{tabular}

Abds abduction strength, ERS external rotation strength, IRS internal rotation strength

* Statistically significant between the pre- and post-operative values $(p<0.05)$

+ Statistically significant between the healed and failed groups $(p<0.05)$ the original footprint without any additional treatment, such as footprint medialisation or muscle advancement. Our findings showed that this procedure achieved good clinical outcomes when the repaired tendon completely healed. Flexion angle and clinical scores significantly improved in the healed group than in the failed group. Even after such surgeries, the prognostic factors for failure were the concomitant SSC lesion and ISP tendon retraction before surgery.

Many studies have reported high failure rates of ARCR for large RCTs [4, 8, 12, 32]. Until 2011, we performed ARCR with a thorough cuff release, footprint medialization [9], margin convergence [2], or autologous patch graft using fascia lata [24] for large RCTs. However, we frequently encountered failure after ARCR for these large RCTs, and a review of our surgical methods was considered necessary. A decision was made to repair the torn cuffs at a tension of $\leq 30 \mathrm{~N}$, in response to Davidson and Rivenburgh's study reporting high-tension repairs $>8 \mathrm{lb}$ [5] $(35.6 \mathrm{~N})$ were associated with poor outcomes. Although some studies have reported using a surgical technique in which over-tension $(\geq 30 \mathrm{~N})$ was not applied to avoid failure [25,36], no study has investigated the failure rate for ARCR in completely repaired cuffs using a tension of $\leq 30 \mathrm{~N}$. Kim et al. measured the tensions needed to repair the cuff of the original footprint and reported a failure rate of $18.2 \%$ after ARCR with single-row repair. They found a significant negative correlation between repair tension and cuff healing [18]. The tension was significantly lesser in the healed group than in the failed group $(26.5 \pm 22.9 \mathrm{~N}$ vs. $37.2 \pm 22.1 \mathrm{~N}$, $p=0.04)$. This report suggested that repairs with tensions $\leq 30 \mathrm{~N}$ could be successfully undertaken. Of the 118 patients in our study, 24 (approximately $20 \%$ ) with a stage III Boileau classification (considered to be large tears) were included. A failure rate of $5.9 \%$ can be considered a relatively good result compared with those reported by previous studies [23, 26, 28, 29, 34].

Generally, several factors have been reported as causes of failure after ARCR, such as age [1,34], tear size $[4,8,12,32]$, tendon quality [17], or muscle FD [16, 29]. Using univariate analysis, Neyton et al. reported that a history of cigarette smoking was a risk factor for failure [26]. Ishitani et al. introduced a novel classification for the tendon stump, graded as the signal intensity compared with that of the deltoid, and reported that type 3 stumps (tendon signal intensity was higher than that of the deltoid) had significantly higher failure rates, using logistic regression analysis [17]. Scheiderer et al. reported a higher critical shoulder angle of $>38^{\circ}$ was a risk factor for failure [28]. We conducted a multivariate regression analysis of the risk factors for cases with failure despite ARCR with tension $\leq 30 \mathrm{~N}$ and found that 
Table 6 Results of univariate analyses between the healed and failed groups

\begin{tabular}{|c|c|c|c|}
\hline & Healed group $(n=111)$ & Failed group $(n=7)$ & $P$-value \\
\hline Age, years & $66.1 \pm 9.2$ & $65.6 \pm 12.9$ & 673 \\
\hline Sex, male: female & 59: 52 & $5: 2$ & .581 \\
\hline Affected side, right: left & 80: 31 & $5: 2$ & .967 \\
\hline Prevalence of DM & $15(+): 96(-)$ & $1(+): 6(-)$ & .952 \\
\hline SSP retraction, Boileau I/II/III & $24 / 65 / 22$ & $1 / 4 / 2$ & .993 \\
\hline ISP retraction, Boileau 0/I/II/III & $77 / 16 / 14 / 4$ & $1 / 4 / 0 / 2$ & $.012^{*}$ \\
\hline Concomitant SSC lesion Lafosse type 0/I/II/III/IV & $44 / 39 / 26 / 1 / 1$ & $2 / 2 / 1 / 1 / 1$ & $.006^{*}$ \\
\hline Concomitant LHB lesion modified Lafosse I/II/III/IVN & $56 / 25 / 12 / 12 / 6$ & $3 / 2 / 0 / 0 / 2$ & 648 \\
\hline Pre $\mathrm{AHI}, \mathrm{mm}$ & $9.41 \pm 1.85$ & $9.35 \pm 2.09$ & .862 \\
\hline Preflex, deg & $125.9 \pm 34.0$ & $139.3 \pm 33.2$ & .194 \\
\hline Pre ER,deg & $56.8 \pm 17.1$ & $56.4 \pm 12.8$ & .758 \\
\hline Pre IR, spine level & Th1 $1.7 \pm 2.9$ & Th12.5 \pm 2.9 & .348 \\
\hline Pre FD of SSC & $61: 38: 7: 5: 0$ & 4:1:0:0:0 & .970 \\
\hline Pre FD of SSP & $25: 63: 20: 3: 0$ & $2: 2: 3: 0: 0$ & 683 \\
\hline Pre FD of ISP & $58: 44: 8: 1: 0$ & $3: 1: 1: 1: 1$ & .252 \\
\hline Pre GFDI & $0.73 \pm 0.51$ & $1.24 \pm 1.13$ & .154 \\
\hline Pre constant score & $48.4 \pm 14.8$ & $54.0 \pm 18.0$ & .502 \\
\hline Pre UCLA score & $14.8 \pm 4.3$ & $17.0 \pm 5.9$ & .360 \\
\hline Pre NRS & $6.6 \pm 1.8$ & $6.8 \pm 2.2$ & .914 \\
\hline
\end{tabular}

* Statistically significant between the healed and failed groups $(p<0.05)$

concomitant SSC lesion and ISP tendon retraction were prognostic factors for failure.

Oh et al. reported that FD of the ISP muscle was the most independent prognostic factor affecting the anatomical outcome [27]. Shimokobe et al. reported that the active ER angle for postero-superior RCTs was a risk factor, using multivariate logistic regression analysis [30]. Their results showed that the loss of the active ER angle correlated with the FD of the ISP muscle. Therefore, the ISP muscle is likely to be associated with post-ARCR failure. In our study, the FD of the ISP muscle was not a prognostic factor for failure, whereas ISP tendon retraction was. To date, ISP tendon retraction as a risk factor has not been reported because it has been challenging to evaluate the ISP tendon retraction degree using conventional MRI. Honda et al. stated that radial-sequence MRI was particularly useful in assessing postero-superior RCTs [14]. Using radial-sequence MRI to evaluate the

Table 7 Results of multivariate regression analyses

\begin{tabular}{|c|c|c|c|c|}
\hline & $\begin{array}{l}\text { Partial } \\
\text { regression } \\
\text { coefficient }\end{array}$ & Odds ratio & $95 \% \mathrm{Cl}$ & $P$-values \\
\hline Age & 0.22 & 1.24 & $0.95-1.64$ & .120 \\
\hline ISP retraction & 2.97 & 19.4 & $1.31-287.3$ & $.031^{*}$ \\
\hline $\begin{array}{l}\text { Concomitant SSC } \\
\text { lesion }\end{array}$ & 1.48 & 4.41 & $1.16-16.8$ & $.030^{*}$ \\
\hline Pre constant score & 0.21 & 1.24 & $0.99-1.55$ & .064 \\
\hline Pre NRS & 0.83 & 2.29 & $0.72-7.31$ & .160 \\
\hline
\end{tabular}

* Statistically significant between the healed and failed groups $(p<0.05)$
RCTs, we identified ISP tendon retraction as a prognostic factor.

Few studies have reported concomitant SSC lesion as a risk factor for failure; however, Herring et al. reported that the number of ruptured tendons correlated with failure, with an odds ratio of 5.83 for three-tendon tears [13]. We consider that even a repaired tendon whose quality and excursion could be considered satisfactory is likely to fail when the concomitant SSC condition is worse because the SSC's superior aspect and SSP's anterior aspect are usually continuous [22].

Whilst our study had certain strengths, it also had some limitations. First, this was a retrospective and nonrandomised study; therefore, selection bias was likely. Second, the tension on the repair must be influenced by the type and correct reposition of the cuff tears and the tendon quality. Although ARCRs were consistently performed in a same arm position under the same anaesthesia, the type of tendons was different in each case, and non-anatomical repairs remaining uncovered footprint were achieved in some cases. In addition, the tendon qualities were not evaluated intraoperatively. These factors may also have influenced the results. Furthermore, the suture bridge repair itself may lead to over-tensioning of the construct compared to single-row repairs. The problem, however, was minimized by uniting all repair techniques to the suture bridge techniques. Third, we performed cuff releases to the maximum extent possible for all cases. However, it is not possible to prove that 
the standardisation was successful before repair. Finally, Kim et al. reported no validated method for measuring the repair tension after repair [18]. Therefore, it is unclear whether the intraoperatively measured values in this study accurately reflect the repair tension. This time, the tension required for complete coverage of the footprint with cuff stump before the repair was regarded as repair tension during the cuff repair, similar to the previous reports $[5,18,25,35,36]$.

\section{Conclusions}

ARCR with suture bridge technique is likely to succeed (failure rate: $5.9 \%$ ) for RCTs that can be extracted using tension $\leq 30 \mathrm{~N}$ before repair. The AHI, ROM except for $\mathrm{ER}$, all functional scores, and isometric muscle strengths improved significantly after complete ARCR. The multivariate regression analysis results indicated that the concomitant SSC lesion and pre-operative ISP tendon retraction were prognostic factors for failure even in ARCR with a tension of $\leq 30 \mathrm{~N}$.

\section{Abbreviations}

RCTs: Rotator cuff tears; ARCR: Arthroscopic rotator cuff repair; SSC: Subscapularis; SSP: Supraspinatus; ISP: Infraspinatus; MRI: Magnetic resonance imaging; LHB: Long head of the biceps brachii; FD: Fatty degeneration; GFDI: Global fatty degeneration index; ROM: Range of motion; $\mathrm{AHI}$ : Acromiohumeral interval; ER: External rotation; IR: Internal rotation; UCLA: University of California, Los Angeles; NRS: Numeric rating score; DM: Diabetes mellitus.

\section{Acknowledgements}

We wish to thank Editage (www.editage.com) for English language editing. We also wish to express our sincere thanks to President Mitsuo Ochi and Professor Yu Mochizuki for their invaluable mentoring and encouragement.

\section{Informed consent}

Informed consent was obtained from all individual participants in this study.

\section{Authors' contributions}

SY contributed substantially to the surgical treatment, the study design, writing and drafting of the manuscript. YN, YH, HN, RM, NM and YS were contributed to helping the surgeries, the data collection, and the statistical analyses. NA mainly directed this manuscript. All authors read and approved the final manuscript prior to submission.

\section{Funding}

None.

\section{Declarations}

Ethics approval and consent to participate

This retrospective study was approved by our Institutional Review Board (permission number: E-2079).

\section{Competing interests}

The authors declare that they have no competing interests.

Received: 23 February 2021 Accepted: 5 March 2021

Published online: 16 March 2021

\section{References}

1. Boileau P, Brassart N, Watkinson D, Carles M, Hatzidakis AM, Krishnan SG (2005) Arthroscopic repair of full-thickness tears of the supraspinatus: does the tendon really heal? J Bone Joint Surg Am 87(6):1229-1240

2. Burkhart SS, Athanasiou KA, Wirth MA (1996) Margin convergence: a method of reducing strain in massive rotator cuff tears. Arthroscopy 12(3):335-338

3. Burkahrt SS, Lo IK (2006) Arthroscopic rotator cuff repair. J Am AcadOrthopSurg 14(6):333-346

4. Cho NS, Rhee YG (2009) The factors affecting the clinical outcome and integrity of arthroscopically repaired rotator cuff tears of the shoulder. Clin Orthop Surg 1(2):96-104

5. Davidson PA, Rivenburgh DW (2000) Rotator cuff repair tension as a determinant of functional outcome. J Shoulder Elbow Surg 9(6):502-506

6. Dierckman BD, Ni JJ, Karzel RP, Getelman MH (2018) Excellent healing rates and patient satisfaction after arthroscopic repair of medium to large rotator cuff tears with a single-row technique augmented with bone marrow vents. Knee Surg Sports Traumatol Arthrosc 26(1):136-145

7. Fuchs B, Weishaupt D, Zanetti M, Hodler J, Gerber C (1999) Fatty degeneration of the muscles of the rotator cuff; assessment by computed tomography versus magnetic resonance imaging. J Shoulder Elbow Surg 8(6):599-605

8. Galatz LM, Ball CM, Teefey SA, Middleton WD, Yamaguchi K (2004) The outcome and repair integrity of completely arthroscopically repaired large and massive rotator cuff tears. J Bone Joint Surg Am 86(2):219-224

9. Ghazanfari A, Henderson DJH, Nourissat G (2017) An arthroscopic humeral medializing repair of the supraspinatus. Arthrosc Tech 6(6):e2211-e2215

10. Gupta AK, Hug K, Berkoff DJ, Boggess BR, Gavigan M, Malley PC et al (2012) Dermal tissue allograft for the repair of massive irreparable rotator cuff tears. Am J Sports Med 40(1):141-147

11. Hakimi O, Mouthuy PA, Carr A (2013) Synthetic and degradable patches: an emerging solution for rotator cuff repair. Int J Exp Pathol 94(4):287-292

12. Henry P, Wasserstein D, Park S, Dwyer T, Chahal J, Slobogean G et al (2015) Arthroscopic Repair for chronic massive rotator cuff tears: a systematic review. Arthroscopy 31(12):2472-2480

13. Herring MJ, White M, Braman JP (2019) The WORC index and predicting treatment failure in patients undergoing primary arthroscopic rotator cuff repair. Orthop J Sports Med 29(7):2325967119859518

14. Honda H, Morihara T, Arai Y, Horii M, Ito H, Furukawa R et al (2015) Clinical application of radial magnetic resonance imaging for evaluation of rotator cuff tear. Orthop Traumatol Surg Res 101(6):715-719

15. Iannotti JP, McCarron J, Raymond CJ, Ricchetti ET, Abboud JA, Brems JJ et al (2010) Agreement study of radiographic classification of rotator cuff tear arthropathy. J Shoulder Elbow Surg 19(8):1243-1249

16. Iijima Y, Matsuki K, Hoshika S, Ueda Y, Hamada H, Tokai M et al (2019) Relationship between postoperative retear and preoperative fatty degeneration in large and massive rotator cuff tears: quantitative analysis using T2 mapping. J Shoulder Elbow Surg 28(8):1562-1567

17. Ishitani E, Harada N, Sonoda Y, Okada F, Yara T, Katsuki I (2019) Tendon stump type on magnetic resonance imaging is a predictive factor for retear after arthroscopic rotator cuff repair. J Shoulder Elbow Surg 28(9):1647-1653

18. Kim DH, Jang YH, Choi YE, Lee HR, Kim SH (2016) Evaluation of repair tension in arthroscopic rotator cuff repair. Does it really matter to the integrity of the rotator cuff? Am J Sports Med 44(11):2807-2812

19. Kim SJ, Lee IS, Kim SH, Lee WY, Chun YM (2012) Arthroscopic partial repair of irreparable large to massive rotator cuff tears. Arthroscopy 28(6):761-768

20. Lafosse L, Lanz U, Saintmard B, Campens C (2010) Arthroscopic repair of subscapularis tear: surgical technique and results. Orthop Traumatol Surg Res 96(8 Suppl):S99-108

21. Lafosse L, Reiland Y, Baier GP, Toussaint B, Jost B (2007) Anterior and posterior instability of the long head of the biceps tendon in rotator cuff tears: a new classification based on arthroscopic observations. Arthroscopy 23(1):73-80

22. Lo IK, Burkhart SS (2003) The comma sign: an arthroscopic guide to the torn subscapularis tendon. Arthroscopy 19(3):334-337 
23. Mihata T, Lee TQ, Watanabe C, Fukunishi K, Ohue TT et al (2013) Clinical results of arthroscopic superior capsule reconstruction for irreparable rotator cuff tears. Arthroscopy 29(3):459-470

24. Mori D, Funakoshi N, Yamashita F, Wakabayashi T (2015) Effect of fatty degeneration of the infraspinatus on the efficacy of arthroscopic patch autograft procedure for large to massive rotator cuff tears. Am J Sports Med 43(5):1108-1117

25. Morihara T, Kida Y, Furukawa R, Sukenari T, Kabuto Y, Kurokawa M et al (2018) Therapeutic outcomes of muscular advancement by an arthroscopic-assisted modified Debeyre-Patte procedure for irreparable large and massive rotator cuff tears. J Orthop Sci 23(3):495-503

26. Neyton L, Godenèche A, Nové-Josserand L, Carrillon Y, Cléchet J, Hardy MB (2013) Arthroscopic suture-bridge repair for small to medium size supraspinatus tear: healing rate and retear pattern. Arthroscopy 29(1):10-17

27. Oh JH, Kim SH, Ji HM, Jo KH, Bin SW, Gong HS (2009) Prognostic factors affecting anatomic outcome of rotator cuff repair and correlation with functional outcome. Arthroscopy 25(1):30-39

28. Scheiderer B, Imhoff FB, Johnson JD, Aglio J, Cote MP, Beitzel K et al (2018) Higher critical shoulder angle and acromion index are associated with increased retear risk after isolated supraspinatus tendon repair at shortterm follow up. Arthroscopy 34(10):2748-2754

29. Shim SB, Jeong JY, Yum TH, Yoo JC (2018) A comparative study to evaluate the risk factors for medium-sized rotator cuff tear in patients younger than 50 years of age. Arthroscopy 34(11):2971-2979

30. Shimokobe H, Gotoh M, Honda H, Nakamura H, Mitsui Y, Kakuma T et al (2017) Risk factors for retear of large/massive rotator cuff tears after arthroscopic surgery: an analysis of tearing patterns. J Orthop Surg Res 12(1):140

31. Spencer EE, Dunn WR, Wright RW, Wolf BR, Spindler KP, McCarty E et al (2008) Interobserver agreement in the classification of rotator cuff tears using magnetic resonance imaging. Am J Sports Med 36(1):99-103

32. Sugaya H, Maeda K, Matsuki K, Moriishi J (2007) Repair integrity and functional outcome after arthroscopic double-row rotator cuff repair. A prospective outcome study. J Bone Joint Surg Am 89(5):953-960

33. Tauro JC (1999) Arthroscopic "interval slide" in the repair of large rotator cuff tears. Arthroscopy 15(5):527-530

34. Voigt C, Bosse C, Vosshenrich R, Schulz AP, Lill H (2010) Arthroscopic supraspinatus tendon repair with suture-bridging technique: functional outcome and magnetic resonance imaging. Am J Sports Med 38(5):983-991

35. Yokoya S, Harada Y, Negi H, Matsushita R, Matsubara N, Adachi N (2020) Arthroscopic rotator cuff repair with muscle advancement and artificial biodegradable sheet reinforcement for massive rotator cuff tears. Orthop J Sports Med 8(10):2325967120960166

36. Yokoya S, Nakamura Y, Harada Y, Ochi M, Adachi N (2019) Outcomes of arthroscopic rotator cuff repair with muscle advancement for massive rotator cuff tears. J Shoulder Elbow Surg 28(3):445-452

37. Yokoya S, Mochizuki Y, Nagata Y, Deie M, Ochi M (2008) Tendon-bone insertion repair and regeneration using polyglycolic acid sheet in the rabbit rotator cuff injury model. Am J Sports Med 36(7):1298-1309

38. Yokoya S, Mochizuki Y, Natsu K, Omae H, Nagata Y, Ochi M (2012) Rotator cuff regeneration using a bioabsorbable material with bone marrowderived mesenchymal stem cells in a rabbit model. Am J Sports Med 40(6):1259-1268

\section{Publisher's Note}

Springer Nature remains neutral with regard to jurisdictional claims in published maps and institutional affiliations. 